\title{
Facile one-pot synthesis of amoxicillin-coated gold nanoparticles and their antimicrobial activity
}

\author{
Marco Demurtas • Carole C. Perry \\ Published online: 4 December 2013 \\ (C) The Author(s) 2013. This article is published with open access at SpringerLink.com
}

\begin{abstract}
Nanomaterials have been the object of intense study due to promising applications in a number of different disciplines. In particular, medicine and biology have seen the potential of these novel materials with their nanoscale properties for use in diverse areas such as imaging, sensing and drug vectorisation. Gold nanoparticles (GNPs) are considered a very useful platform to create a valid and efficient drug delivery/carrier system due to their facile and well-studied synthesis, easy surface functionalization and biocompatibility. In the present study, stable antibiotic conjugated GNPs were synthesised by a one-step reaction using a poorly water soluble antibiotic, amoxicillin. Amoxicillin, a member of the penicillin family, reduces the chloroauric acid to form nanoparticles and at the same time coats them to afford the functionalised nanomaterial. A range of techniques including UV-vis spectroscopy, dynamic light scattering (DLS), transmission electron microscopy (TEM) and thermogravimetric analysis (TGA) were used to ascertain the gold/drug molar ratio and the optimum temperature for synthesis of uniform monodisperse particles in the ca. $30-40 \mathrm{~nm}$ size range. Amoxicillin-conjugated gold showed an enhancement of antibacterial activity against Escherichia coli compared to the antibiotic alone.
\end{abstract}

Keywords Gold · Nanoparticles · Amoxicillin .

Escherichia coli $\cdot$ Antibacterial activity

\section{Introduction}

Since the discovery of the first antibiotic [1], the scientific community has spent a great amount of effort in studying,

M. Demurtas $\cdot$ C. C. Perry $(\bowtie)$

Interdisciplinary Biomedical Research Centre, School of Science and

Technology, Nottingham Trent University, Clifton Lane,

Nottingham NG11 8NS, UK

e-mail: carole.perry@ntu.ac.uk developing and synthesising new types of antibiotic to fight bacteria. A large number of antibiotics, divided into various families having structural or functional similarity, have been discovered as a result of this intensive research. Unfortunately, the extensive use of these antibiotics has caused bacteria to develop new resistance mechanisms including prevention of drug interaction with the target, efflux of the antibiotic from the cell and direct destruction or modification of the compound $[2,3]$. To win the battle against new strains of bacteria, an alternative approach may be to use the already well-studied drug and conjugate it with other entities in order to enhance anti-microbial activity. In the last decade, nanomaterials have been widely used in biotechnology [4]. In particular, drugconjugated gold nanoparticles (GNPs) have been intensively investigated as delivery/carrier systems [5-7]. GNPs are characterised by properties that are potentially suitable for use in this area. First, gold nanoparticles can be synthesised in a controlled fashion affording a wide range of sizes $(1-150 \mathrm{~nm})$ [5]. Also, the surface of the gold nanoparticles can be functionalised through covalent or non-covalent interaction without structural modification of the drug [8]. The conjugation of a drug with the inorganic particles could increase the bio-stability, the bio-distribution and also suppress some side effects [5-7]. In addition, the high surface area typical of nanoparticles guarantees a huge drug load; for example, Zubarev et al. were able to couple $\approx 70$ molecules of paclitaxel to a GNP of $2 \mathrm{~nm}$ [5]. Several examples are available in the literature of the anti-microbial activity of antibiotics conjugated with gold nanoparticles [9-11]. Usually, the synthetic pathway starts with the reduction of a chloroauric acid solution via a range of methods [12-15]. Historically, Turkevich et al. reported [12] the production of a gold colloid solution using sodium citrate as reducing and stabilising agent in 1951. In 1973, Frens et al. showed [13] the possibility of controlling the gold nanoparticle size by changing the molar ratio of the reagents. In 1994, Brust et al. proposed [14] a novel method to produce stable gold nanoparticles using a two-phase technique with $\mathrm{NaBH}_{4}$ as the 
reducing agent. In this method, chloroauric acid is transferred to toluene using tetraoctylammonium bromide as the phasetransfer reagent and the gold reduced by $\mathrm{NaBH}_{4}$ in the presence of dodecanethiol. Following the formation of the gold nanoparticles, their functionalization involves one or more reaction steps in order to attach the drug to the particle surface. Various approaches of functionalization have been proposed in the literature [5-7].

Recently, we reported a simpler water-based approach to antibiotic-coated gold particles [16] where cefaclor, a second generation antibiotic from the cephalosporin family, was shown to act as a reducing agent to produce the gold nanoparticles and, at the same time, coat them affording the desired anti-microbial nanomaterial, all in a single synthesis step. We were able to control the particle size, the amount of drug loaded and the production time just by changing reaction temperature. Gold nanoparticles alone did not show any antibacterial property, and we were able to show that conjugation between the particles and the drug was necessary to enhance antibacterial activity against common gram-positive and gram-negative bacteria compared to cefaclor alone [16]. Moreover, we were able to coat a functionalised glass slide with cefaclor-reduced gold nanoparticles creating an antimicrobial film which was very robust and inhibited the growth of Escherichia coli on the surface over a range of $\mathrm{pH}$ conditions designed to mimic common cleaning scenarios [16].

Starting from these results, we wanted to investigate the possibility of using this one-pot synthesis with other drugs, including antibiotics. The idea was to demonstrate that the one-step synthesis with cefaclor was not an isolated case, and that this approach, where the desired drug acts as reducing and capping agent, can be used to create a wide range of drugcoated GNPs. We identified various possible drug candidates that share some features with cefaclor, such as chemical structure and more importantly, the presence of a primary amine, which we demonstrated [16] to be at least partly responsible for the reducing/capping actions in the reaction. Different molecules from the cephalosporin and penicillin families, both $\beta$-lactam drugs, were investigated, and among them, amoxicillin, an antibiotic having poor water solubility, was chosen. Amoxicillin (Fig. 1) is a broad-spectrum $\beta$ lactam antibiotic used to treat bacterial infections of the chest, urine or ear [17]. Amoxicillin is effective against a range of bacteria inhibiting the synthesis of the bacterial wall cell, and it is widely used because it is better adsorbed compared with other similar antibiotics [17]. Herein, we describe the singlestep synthesis of amoxicillin-coated gold nanoparticles, optimization of the reaction and its antimicrobial activity against E. coli. Also, we prove that an antibiotic, with very limited water solubility such as amoxicillin, is suitable for the one-pot synthesis without decreasing the antibacterial properties and bio-distribution.

\section{Experimental}

Materials

$\mathrm{HAuCl}_{4}$ and amoxicillin were purchased from Sigma-Aldrich and used as received. Cellulose dialysis tubing $(10 \mathrm{kDa})$ was purchased from Spectrum Laboratory Inc. and the membrane prepared for use by removing any glycerine and preservative according to the manufacturers procedure. E. coli culture (K12) was used for the antibacterial study.

Synthesis and characterization of amoxicillin-coated gold nanoparticles

Aqueous solutions of amoxicillin ( 0.01 (not fully solubilised), $0.001 \mathrm{M})$ and $\mathrm{HAuCl}_{4}(0.01 \mathrm{M})$ were prepared for use in the one-pot synthesis according to our previous work [16]. Materials were prepared at a range of gold/antibiotic molar ratios and temperatures $\left(20-70{ }^{\circ} \mathrm{C}\right)$. An example preparation (as for the material used for antimicrobial activity testing) is as follows: $9.65 \mathrm{~mL}$ of dd. $\mathrm{H}_{2} \mathrm{O}, 100 \mu \mathrm{L}$ of $\mathrm{HAuCl}_{4}(0.01 \mathrm{M})$ and $250 \mu \mathrm{L}$ of amoxicillin $(0.001 \mathrm{M})$ were mixed and the resulting solution heated in a water bath at $40{ }^{\circ} \mathrm{C}$ for $4 \mathrm{~h}$. Freshly synthesised amoxicillin-coated gold nanoparticles were dialysed using a $10 \mathrm{kDa}$ cut-off cellulose membrane against double-distilled water for $24 \mathrm{~h}$ to remove any unreacted chemicals. Once dialysed, the samples were freeze-dried at $223 \mathrm{~K}$ using a VirTis freeze dryer.

The shape and size of the gold nanoparticles was determined by transmission electron microscopy (TEM) (JEOL 2010; accelerating voltage of $200 \mathrm{kV}$ ). TEM samples were prepared by depositing a $10 \mu \mathrm{l}$ drop of drug-coated gold nanoparticle solution on a carbon-coated grid (Quantifoil grids: $\mathrm{Cu} 200$ mesh). Samples were air-dried for 5-10 min and excess solution removed using a tissue paper before being left to dry at room temperature overnight. One hundred particles were measured and their average size computed. The size and the charge carried by the gold and gold/antibiotic nanoparticles in solution was also determined by dynamic light scattering/zeta potential measurement (DLS) using a Malvern Instrument Nano-S.

The antibiotic 'load' of the nanoparticles was determined using thermogravimetric analysis (TGA) using a MettlerToledo (TGA/SDTA851) instrument. Samples were heated in air over a temperature range of $30-900{ }^{\circ} \mathrm{C}$, at $10^{\circ} \mathrm{C} / \mathrm{min}$.

Antimicrobial activity testing

E. coli was grown in lysogeny broth (LB) for $24 \mathrm{~h}$ at $37{ }^{\circ} \mathrm{C}$ and cell counts were quantified by $\mathrm{OD}_{590}$ measurement. Amox-GNPs solutions were added to E. coli suspensions, incubated and shaken at $37{ }^{\circ} \mathrm{C}$ prior to dilution, plating and incubation on LB Agar plates. Antibacterial activity was 


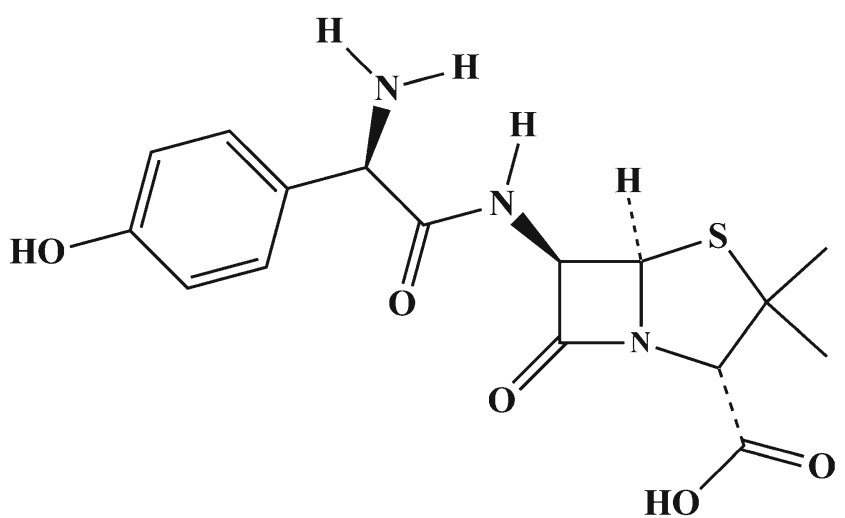

Fig. 1 Chemical structure of amoxicillin

determined by plotting colony-forming units against incubation times 0,2 and $4 \mathrm{~h}$. For each experiment, three different Amox-GNPs solutions were added to a suspension of bacteria $(100,200$ and $300 \mu \mathrm{g} / \mathrm{mL})$, which corresponded to 33,66 and $99 \mu \mathrm{g} / \mathrm{mL}$ of amoxicillin present on the GNPs, respectively. Also, a solution containing amoxicillin alone $(150 \mu \mathrm{g} / \mathrm{mL})$ was prepared as a control to compare the antibacterial behaviour. The microbiology experiments were performed in triplicate.

Colony counting was performed using a Synbiosis aCOLyte $(7510 / \mathrm{SYN})$ colony counter. The minimum inhibition concentration was calculated according to the method used by Sambhy et al. [18]

\section{Results and discussion}

Amoxicillin-coated gold nanoparticles were prepared according to the one-pot synthesis of cefaclor-GNPs presented in our previous work [16]. In this case, the molar ratio of the reagents was varied, in part due to the lower water solubility of amoxicillin. A complete UV-vis study was carried out in order to establish the best reaction conditions to achieve the coated gold nanoparticles. Three properties (peak position, peak breadth and peak intensity) of the UV-vis spectrum were used. The surface plasmon resonance (SPR) band typical of the gold nanoparticles lies in the visible region of the electromagnetic spectrum with any changes to the surroundings of these particles, such as surface modification and aggregation, leading to a colorimetric change of the dispersion. Thus, the SPR peak position provides information about the particle size and the broadness of the SPR absorption peak information on particle size distribution, a broad band signifying a broad particle size distribution and aggregation. Finally, the absorption value is proportional to the amount of gold nanoparticles produced $[19,20]$. Figure 2a shows the UV-vis spectra of amoxicillin-GNPs samples produced using four different molar ratios of $\mathrm{Au}(\mathrm{IIII} / \mathrm{amoxicillin}$. The best result was obtained with a molar ratio of $4: 1$ as confirmed by a sharp peak in
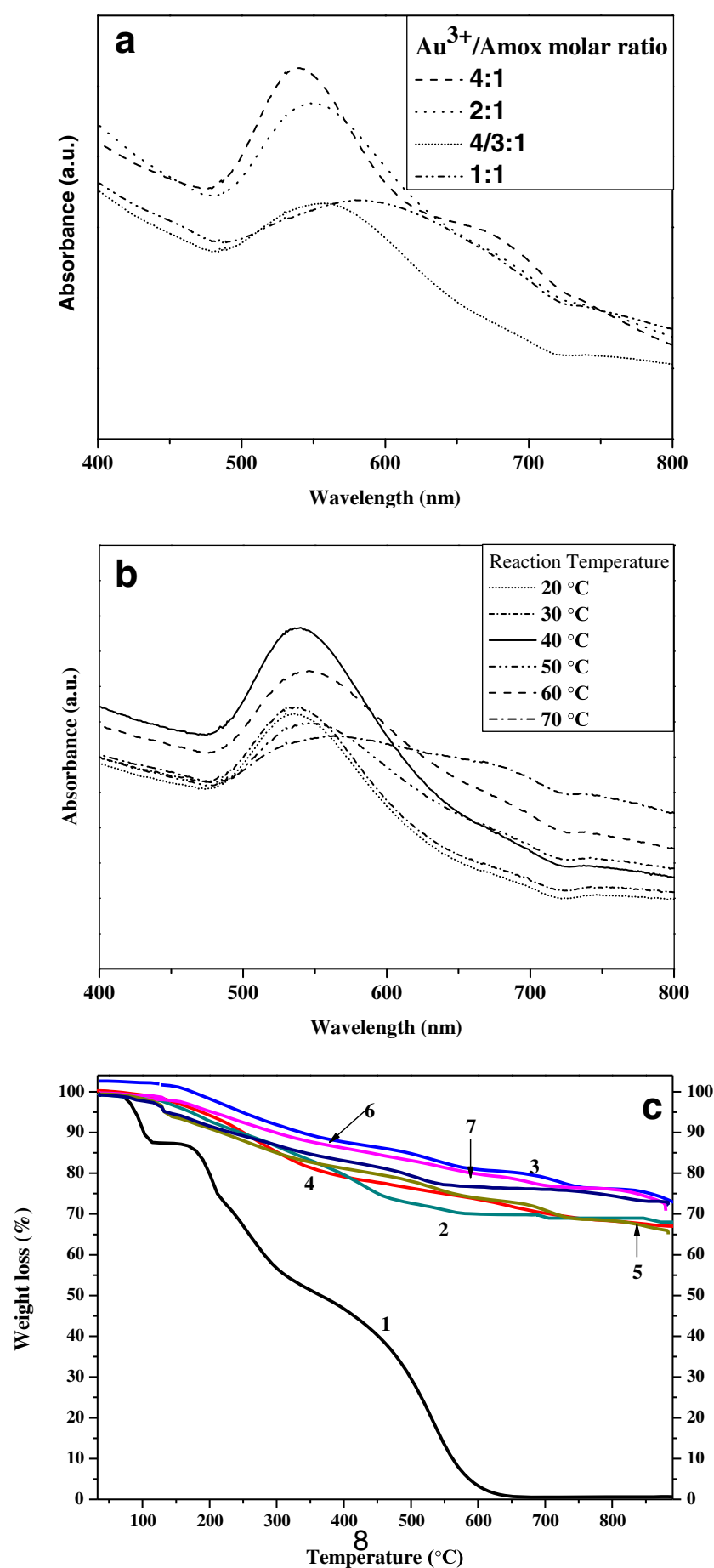

Fig. 2 a UV-vis spectra of amoxicillin-coated gold nanoparticles obtained at different molar ratios of $\mathrm{Au}(\mathrm{III})$ and amoxicillin. b UV-vis spectra of amoxicillin-coated gold nanoparticles synthesised at different temperatures (gold: antibiotic molar ratio of 4:1). c TGA analyses of amoxicillin-coated gold nanoparticles synthesised at different temperatures with the same molar ratio as in $1 \mathrm{~b}$. Amoxicillin (1), amoxicillinGNPs at $20^{\circ} \mathrm{C}(2), 30^{\circ} \mathrm{C}(3), 40{ }^{\circ} \mathrm{C}(4), 50{ }^{\circ} \mathrm{C}(5), 60^{\circ} \mathrm{C}(6)$ and $70{ }^{\circ} \mathrm{C}(7)$

Fig. 2a. Our previous work [16] showed the importance of temperature on the one-pot synthesis, where for cefaclor increasing reaction temperature led to a decrease in reaction 


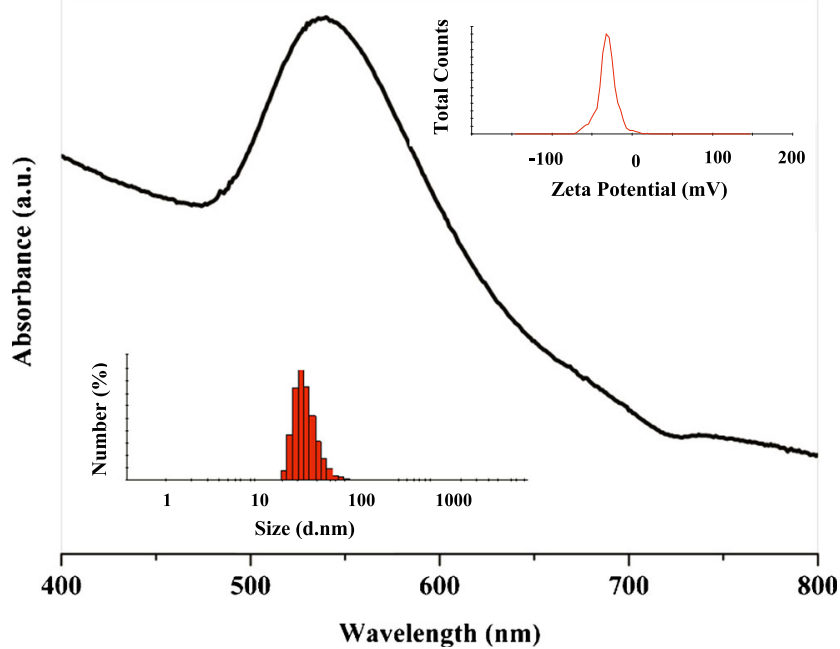

Fig. 3 UV-vis spectrum of amoxicillin-conjugated gold nanoparticles synthesised at $40{ }^{\circ} \mathrm{C}$. Histogram of size distribution (inset bottom left) and zeta-potential measurement (inset top right) of the amoxicillin-conjugated gold nanoparticles obtained at $40^{\circ} \mathrm{C}$ for the $4: 1$ gold: antibiotic sample

time, a linear decrease in particle size and decrease in amount of drug loaded on the nanoparticles [16]. In the current study, a linear dependence of particle size with temperature was not seen; rather, the UV-vis spectra of the resulting gold colloid solutions presented in Fig. 2b show that temperature largely affected the growth and aggregation of the particles with the best (least aggregated/more uniform in size) particles being obtained at $40{ }^{\circ} \mathrm{C}$.

With the optimum synthesis conditions, an aqueous solution of amoxicillin and chloroauric acid (1:4) heated at $40{ }^{\circ} \mathrm{C}$ in a water bath for $4 \mathrm{~h}$ gave the typical red wine colour of a gold colloid solution. The UV-vis absorption spectrum in Fig. 3 shows the characteristic SPR band with the maximum peak at $540 \mathrm{~nm}$. The sharp absorption band is evidence of a fine nanoparticle dispersion without aggregation and precipitation (flocculation). Zeta potential measurements performed

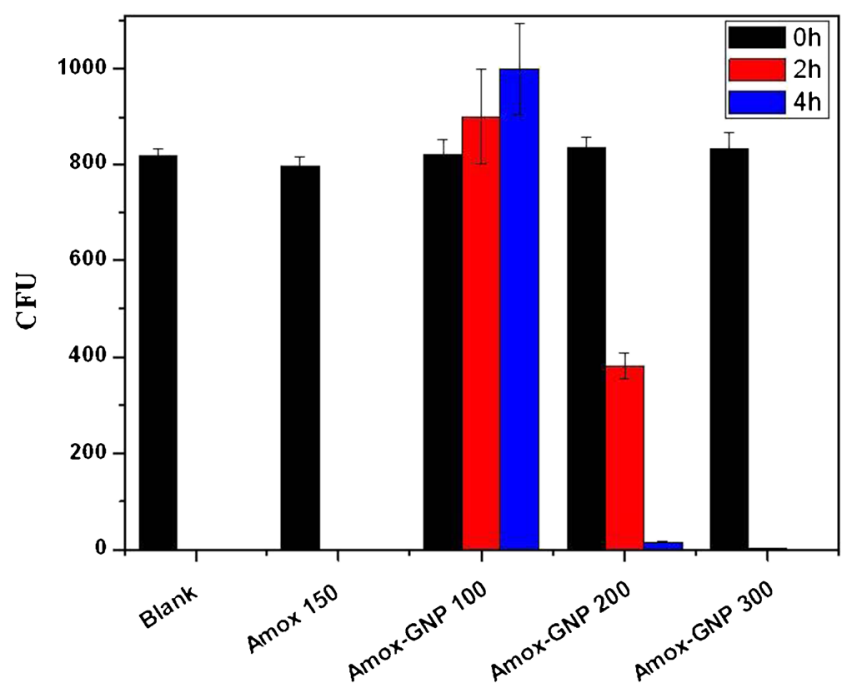

Fig. 5 Histogram plot showing antimicrobial activity of amoxicillinconjugated gold nanoparticles (synthesis as per Fig. 2) and amoxicillin on $E$. coli after different incubation times ( 0,2 , and 4 h). All concentrations are in units of $\mu \mathrm{g} / \mathrm{ml}$. The 'blank' without addition of amoxicillin or amoxicillin-conjugated gold nanoparticles is shown for $t=0$ only where it was possible to measure the colony forming units

on the same sample confirmed the stability of the negatively charged amoxicillin-coated gold nanoparticles with a value of $-31.3 \mathrm{mV}$. TEM analysis (Fig. 4) confirmed the uniform size and particle distribution with an average spherical diameter of $32.61 \pm 4.78 \mathrm{~nm}$. The lighter areas surrounding the ca. $30 \mathrm{~nm}$ gold-antibiotic nanoparticles also contain gold in the form of much smaller nanoparticles of the order of 1-2 $\mathrm{nm}$ in diameter, also associated with organic material (antibiotic) that are not discernible separately in the images shown. DLS analysis corroborated this finding with a measured particle size of $37.85 \pm 13.20 \mathrm{~nm}$, Fig. 3 inset. The amount of antibiotic bound to the particles as measured by TGA, Fig. 2c, was around $33 \%$ by weight.
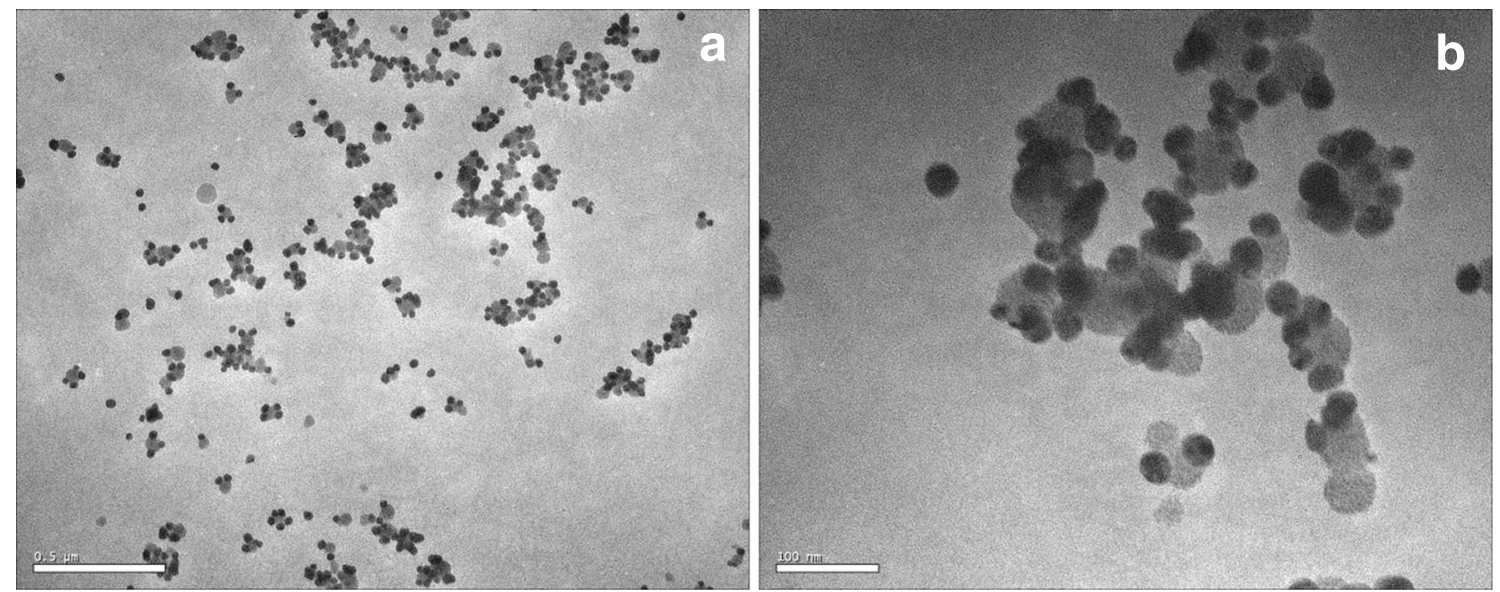

Fig. 4 TEM images of the coated gold nanoparticles synthesised at a 4:1 M ratio of gold: antibiotic at $40{ }^{\circ} \mathrm{C}$ recorded at different magnifications. Scale bar in TEM images is 500 (a) and $100 \mathrm{~nm}$ (b) 
The antimicrobial activity of the antibiotic-coated nanoparticles was determined against a common gramnegative bacteria $E$. coli. Heating to $40{ }^{\circ} \mathrm{C}$ during the formation of the antibiotic loaded particles did not affect the antibacterial capability of the amoxicillin present on the gold nanoparticles.

Figure 5 showed that the lowest concentration sample Amox-GNPs $100 \mu \mathrm{g} / \mathrm{mL}$ did not inhibit the growth of $E$. coli colonies over $4 \mathrm{~h}$ of incubation at $37{ }^{\circ} \mathrm{C}$. The presence of $33 \mu \mathrm{g} / \mathrm{mL}$ of amoxicillin associated with the gold particles was enough to slow down the growth of the gram-negative bacteria. The Amox-GNPs $200 \mu \mathrm{g} /$ $\mathrm{mL}$ sample killed half of the E. coli colonies in $2 \mathrm{~h}$ of incubation, and after $4 \mathrm{~h}$, only $2 \%$ of the number was observed. The $300 \mu \mathrm{g} / \mathrm{mL}$ Amox-GNPs $(99 \mu \mathrm{g} / \mathrm{mL}$ of amoxicillin) sample killed $99 \%$ of the bacteria in $2 \mathrm{~h}$, showing the same antibacterial activity compared to amoxicillin alone when the antibiotic alone was used at a higher concentration $(150 \mu \mathrm{g} / \mathrm{mL})$. The conjugation of the drug with gold nanoparticles enhanced the antimicrobial activity of the antibiotic. The minimum time required to inhibit the complete growth of $E$. coli was $2 \mathrm{~h}$, and the minimum inhibition concentration of gramnegative bacteria obtained from the amoxicillin-reduced gold nanoparticles was determined according to the method of Sambhy et al. [18] as $300 \mu \mathrm{g} / \mathrm{mL}(99 \mu \mathrm{g} /$ $\mathrm{mL}$ of amoxicillin).

\section{Conclusions}

In summary, we describe an easy way to produce antibiotic-coated gold nanoparticles using a single reaction step where the amoxicillin reduced the gold ions and capped the resulting gold nanoparticles. We show that this particular synthetic pathway can be used with antibiotics that are poorly soluble in aqueous solution and that preparation of the gold-coated particles at temperatures above body temperature for extended times does not destroy the activity of the antibiotic. The conjugation of amoxicillin with gold nanoparticles enhances antibacterial activity as the same microbiological activity is achieved with ca. $33 \%$ less amoxicillin. Currently, we are investigating the possibility to use this one-pot synthesis to coat gold nanoparticles with other families of antibiotics and/or drugs, which must display a primary amine group, but also have enough reducing power to generate gold nanoparticles.

Acknowledgments We gratefully acknowledge support for this research from the World Gold Council.
Open Access This article is distributed under the terms of the Creative Commons Attribution License which permits any use, distribution, and reproduction in any medium, provided the original author(s) and the source are credited.

\section{References}

1. Fleming A (1929) On the antibacterial action of cultures of a Penicillium, with special reference to their use in the isolation of $B$. influenzae. Br J Exp Pathol 10(3):226-236

2. Kumar A, Schweizer HP (2005) Bacterial resistance to antibiotics: active efflux and reduced uptake. Adv Drug Deliv Rev 57:1486-1513

3. Neu HC (1992) The crisis in antibiotic resistance. Science 257:1064-1073

4. De M, Ghosh PS, Rotello VM (2008) Applications of nanoparticles in biology. Adv Mater 20(22):4225-4241

5. Ghosh P, Han G, De M, Kim CK, Rotello VM (2008) Gold nanoparticles in delivery applications. Adv Drug Deliv Rev 60(11):1307-1315

6. Duncan B, Kim CK, Rotello VM (2010) Gold nanoparticle platforms as drug and biomacromolecule delivery systems. J Control Release 148(1):122-128

7. Pissuwan D, Niidome T, Cortie MB (2011) The forthcoming applications of gold nanoparticles in drug and gene delivery systems. J Control Release 149(1):65-71

8. Sperling RA, Gil PR, Zhang F, Zanella M, Parak WJ (2008) Biological applications of gold nanoparticles. Chem Soc Rev 37: 1896-1908

9. Kim CK, Ghosh P, Rotello VM (2009) Multimodal drug delivery using gold nanoparticles. Nanoscale 1:61-67

10. Gu H, Ho PL, Tong E, Wang L, Xu B (2003) Presenting vancomycin on nanoparticles to enhance antimicrobial activities. Nano Lett 3(9): 1261-1263

11. Gibson JD, Khanal BP, Zubarev ER (2007) Paclitaxel-functionalized gold nanoparticles. J Am Chem Soc 129(37):11653-11663

12. Turkevich J, Stevenson PC, Hillier J (1951) A study of the nucleation and growth processes in the synthesis of colloidal gold. Faraday Soc 11:55-75

13. Frens $G$ (1973) Controlled nucleation for the regulation on particle size in monodisperse gold suspension. Nature Phys Sci 241(105):20-22

14. Brust M, Walker M, Bethell D, Schiffrin DJ, Whyman R (1994) Synthesis of thiol-derivatised gold nanoparticles in a two-phase Liquid-Liquid system. J Chem Soc Chem Commun 7:801-802

15. Martin MN, Basham JI, Chando P, Eah SK (2010) Charged gold nanoparticles in non-polar solvents: 10-min synthesis and 2D selfassembly. Langmuir 26(10):7410-7417

16. Rai A, Prabhune A, Perry CC (2010) Antibiotic-mediated synthesis of gold nanoparticles with potent antimicrobial activity and their application in antimicrobial coatings. J Mater Chem 20:6789-6798

17. Holten KB, Onusko EM (2000) Appropriate prescribing of oral betalactam antibiotics. Am Fam Physician 62(3):611-620

18. Sambhy V, MacBride MM, Peterson BR, Sen A (2006) Silver bromide nanoparticle/polymer composites: dual action tunable antimicrobial materials. J Am Chem Soc 128:9798-9808

19. Daniel MC, Astruc D (2004) Gold nanoparticles: assembly, supramolecular chemistry, quantum-size-related properties, and applications toward biology, catalysis, and nanotechnology. Chem Rev 104: 293-346

20. Boisselier E, Astruc D (2009) Gold nanoparticles in nanomedicine: preparations, imaging, diagnostics, therapies and toxicity. Chem Soc Rev 28:1759-1782 